\title{
Novel keratin 5 mutation in a family with epidermolysis bullosa simplex
}

\author{
JIAJIA GAO, XUEBIN WANG, FANG ZHENG, SUFANG DONG and XUEPING QIU \\ Center for Gene Diagnosis, Zhongnan Hospital of Wuhan University, Wuhan, Hubei 430071, P.R. China
}

Received October 8,2014; Accepted September 24, 2015

DOI: 10.3892/etm.2015.2811

\begin{abstract}
The aim of the present study was to identify the causative gene defects associated with epidermolysis bullosa simplex (EBS) in a pedigree. The diagnosis of EBS was confirmed in two patients from that pedigree based on the clinical manifestations, histopathological examination of the skin and family history. Blood samples were collected from 6 family members and 100 heathy controls, and genomic DNA and RNA were extracted. Mutation analysis of the keratin 5 gene (KRT5) was conducted using polymerase chain reaction (PCR) direct sequencing and PCR-restriction fragment length polymorphism. In the pedigree, the results of PCR direct sequencing revealed a heterozygous missense mutation in codon 202 of exon 2 of KRT5 (c.605T>A), which led to an amino acid change (p.L202Q) in the patients with EBS but was absent from the unaffected family members and 100 population controls. In conclusion, a novel missense mutation in the KRT5 gene was identified that had a pathogenic role in EBS in the population studied, which enriches the germline mutation spectrum of the KRT5 gene.
\end{abstract}

\section{Introduction}

Epidermolysis bullosa (EB) is a term that describes a group of inherited skin diseases that are characterized by blistering of the skin caused by mechanical trauma (1). EB has been classified into three major subtypes on the basis of the level of the dermoepidermal separation within the basement membrane zone (1). Epidermolysis bullosa simplex (EBS) comprises the most common subtype of $\mathrm{EB}$, which results from tissue separation within the epidermal basal keratinocytes. The worldwide prevalence of EBS has been estimated to be between 1 in 30,000 and 1 in 50,000 (1).

Correspondence to: Dr Fang Zheng, Center for Gene Diagnosis, Zhongnan Hospital of Wuhan University, 169 Donghu Road, Wuchang, Wuhan, Hubei 430071, P.R. China

E-mail: zhengfang@whu.edu.cn

Key words: epidermolysis bullosa simplex, keratin 5, screening mutation
EBS can be subdivided into three major subtypes: i) The mildest subtype, localized EBS (EBS-loc, OMIM 131800), characterized by blistering confined to the hands and feet; ii) the moderately severe variant, generalized EBS (EBS-gen; OMIM 131900), characterized by more generalized blistering, and iii) the most severe variant, the Dowling-Meara type (EBS-DM; OMIM 131760) characterized by severe herpetiform blistering (2).

EBS is mostly caused by a single mutation in either of the keratin genes, keratin 5 (KRT5) or KRT14, inherited in an autosomal dominant fashion $(3,4)$. The majority of mutations are nucleotide substitutions that lead to missense mutations. The KRT5 and KRT14 genes encode the intermediate filament cytoskeleton proteins in basal keratinocytes, which maintain the mechanical integrity of the epidermis (5). Each of these two keratin proteins comprises a central $\alpha$-helical rod domain of 310 amino acids, which consists of four segments (1A, 1B, $2 \mathrm{~A}$ and $2 \mathrm{~B}$ ) and is interrupted by three nonhelical linkers (L1, L12 and L2) (5).

Mutation screening of KRT5 and KRT14 in an EBS proband and their family members could potentially help to improve the understanding of the pathogenesis of EBS and the mutation spectrum of Chinese patients with EBS. In the present study, Chinese patients with EBS were examined for KRT5 germline mutations.

\section{Patients and methods}

Patients and pedigree. In total, 6 members of the EBS pedigree (Fig. 1) were enrolled in the study in October 15th, 2013. The members II:6 (female, 46-years-old) and III:3 (male, 23 -years-old) were diagnosed with EBS and treated in the Zhongnan Hospital of Wuhan University (Wuhan, China). The diagnosis was based on the clinical features, family history and histopathological examination of the skin of the patients. The clinical records, family history and results from the histopathological examination of the patients were collected.

The proband presented with blistering after mechanical trauma, which occurred mainly on the feet or hands following exercise and the symptoms deteriorated in the summer. The bullae healed without scarring. All four affected members in the pedigree had the same clinical manifestations as the proband. Analyses also included 100 unaffected control subjects. 
Table I. Primers and conditions for keratin 5 amplification by reverse transcription-polymerase chain reaction.

\begin{tabular}{lllcc}
\hline & \multicolumn{2}{c}{ Primers $\left(5^{\prime} \rightarrow 3^{\prime}\right)$} & Product & $\begin{array}{c}\text { Annealing } \\
\text { Exon }\end{array}$ \\
\cline { 2 - 5 } & \multicolumn{1}{c}{ Forward } & Reverse & sp $)$ & temperature $\left({ }^{\circ} \mathrm{C}\right)$ \\
\hline $2,3,4,5,6$ (for cDNA) & CGGTAGTGGATTTGGTTTCG & TGGTGTTGCGGAGGTCAT & 845 & 56 \\
7,8 (for cDNA) & CGATGACCTCCGCAACA & CTCCTGGGAACCAAAGAAT & 798 & 57 \\
2 (for DNA) & AGAGGGACGGAAAGAGG & CAAAGGAAGGCATGGTAG & 401 & 59 \\
\hline
\end{tabular}

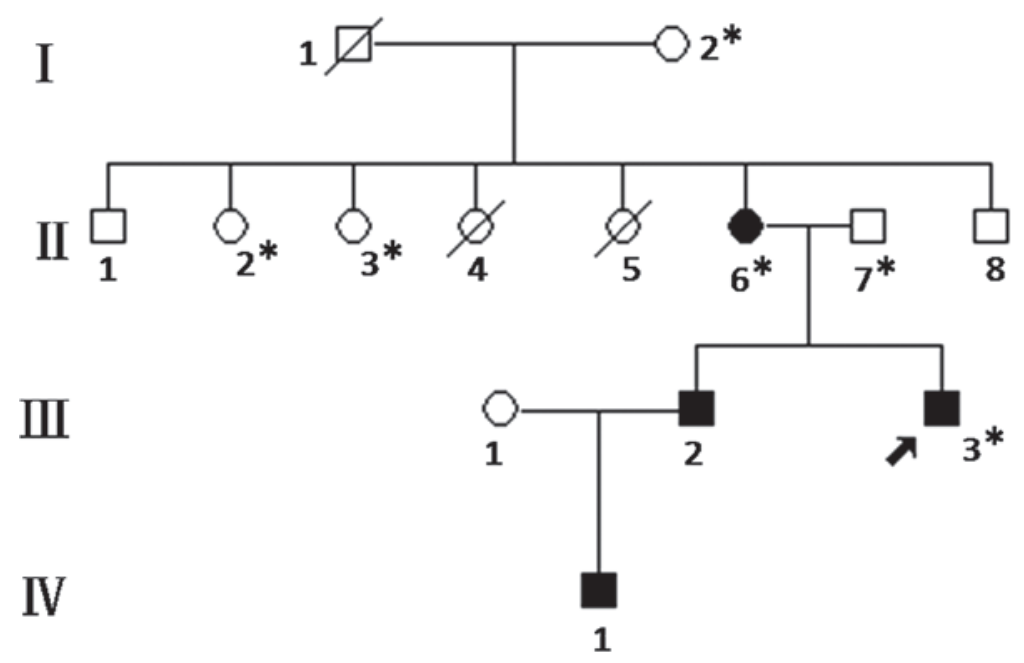

Figure 1. Family pedigree. The family history revealed four affected members across four generations. The dark symbols represent the affected members of the family, while the unfilled symbols indicate the unaffected members. Circles represent female and squares male family members. Asterisks indicate the patients who participated in the present study. The arrow indicates the proband, while strikes (/) through the symbols represent deceased members.

The study was approved by the Zhongnan Hospital Research Ethics Committee and was conducted in accordance with the Declaration of Helsinki. Informed consent was obtained from all individuals prior to their participation in the study.

Mutational screening. Approximately 4-5 $\mathrm{ml}$ peripheral venous blood was drawn from the family members and 100 population controls. Genomic DNA was extracted using the standard sodium dodecyl sulfate-proteinase K method (6), quantified using a spectrophotometer (Nanodrop 2000; Thermo Fisher Scientific, Inc., Waltham, MA, USA) at $260 \mathrm{~nm}$ and stored at $-20^{\circ} \mathrm{C}$ until use. The total RNA sample of the proband was extracted using the ComWin RNA extraction kit (Beijing ComWin Biotech Co., Ltd., Beijing, China) according to the manufacturer's instructions. Reverse transcription (RT) was performed with $1 \mu \mathrm{g}$ total RNA using a Revert First Strand cDNA Synthesis kit (Thermo Scientific, Inc., Waltham, MA, USA). Specific primers for KRT5 were designed using Primer 3, version 0.4.0 (http://frodo.wi.mit.edu/primer3/) (sequences listed in Table I) and synthesized by Qingke Biotechnology Co., Ltd. (Wuhan, China). DNA or cDNA fragments were amplified using PCR tubes containing $10 \mathrm{pmol}$ of each primer, $1.5 \mathrm{mmol} / \mathrm{Mg}^{2+}, 2.0 \mathrm{mmol} / 1 \mathrm{dNTP}, 1.0$ units Taq DNA polymerase (Fermentas; Thermo Fisher Scientific, Inc.) and $100 \mathrm{ng}$ DNA. The amplification was performed as follows: Initial denaturation at $95^{\circ} \mathrm{C}$ for $10 \mathrm{~min}$ followed by 35 cycles of denaturation at $95^{\circ} \mathrm{C}$ for $30 \mathrm{sec}$, annealing at 56,57 or $59^{\circ} \mathrm{C}$ (as specified in Table I) for $30 \mathrm{sec}$, extension at $72^{\circ} \mathrm{C}$ for $1 \mathrm{~min}$ and final extension at $72^{\circ} \mathrm{C}$ for $10 \mathrm{~min}$. Amplifications were performed in a Hema 9600 PCR thermocycler (Hema Medica Instrument Co., Ltd., Zhuhai, China). PCR-amplified fragments were purified and sequenced by Qingke Biotechnology Co., Ltd. using an Applied Biosystems Genetic Analyzer 3730xl (Thermo Fisher Scientific, Inc.).

Mutation analysis was conducted using the PCR-restriction fragment length polymorphism (PCR-RFLP) method in 6 family members and 100 population controls. The PCR products $(8 \mu \mathrm{l})$ were digested with $E c o$ NI restriction enzyme (New England Biolabs Inc., Ipswich, MA, USA) at $37^{\circ} \mathrm{C}$ overnight. The resulting fragments were separated by electrophoresis on a $2 \%$ agarose gel (Biowest, Madrid, Spain) for $20 \mathrm{~min}$ with $100 \mathrm{~V}$ in $0.5 \mathrm{X}$ Tris/borate/EDTA buffer (Sheng Gong Biotechnology Co., Ltd., Shanghai, China). Subsequently, the fragments were stained by goldview (Sai Baisheng Biotechnology Co., Ltd., Shanghai, China) were visualized under a UV transilluminator (Bio-Rad Laboratories, Inc., Hercules, CA, USA).

Protein-protein Basic Local Alignment Search Tool (BLAST) of KRT5. The protein BLAST program (http://blast.ncbi.nlm. nih.gov/Blast.cgi) was used to align the proteins of different species. The protein sequences of keratin were inserted in the pblast frame, and the proteins of different species (such as human, dog, mouse and rat) were determined. 


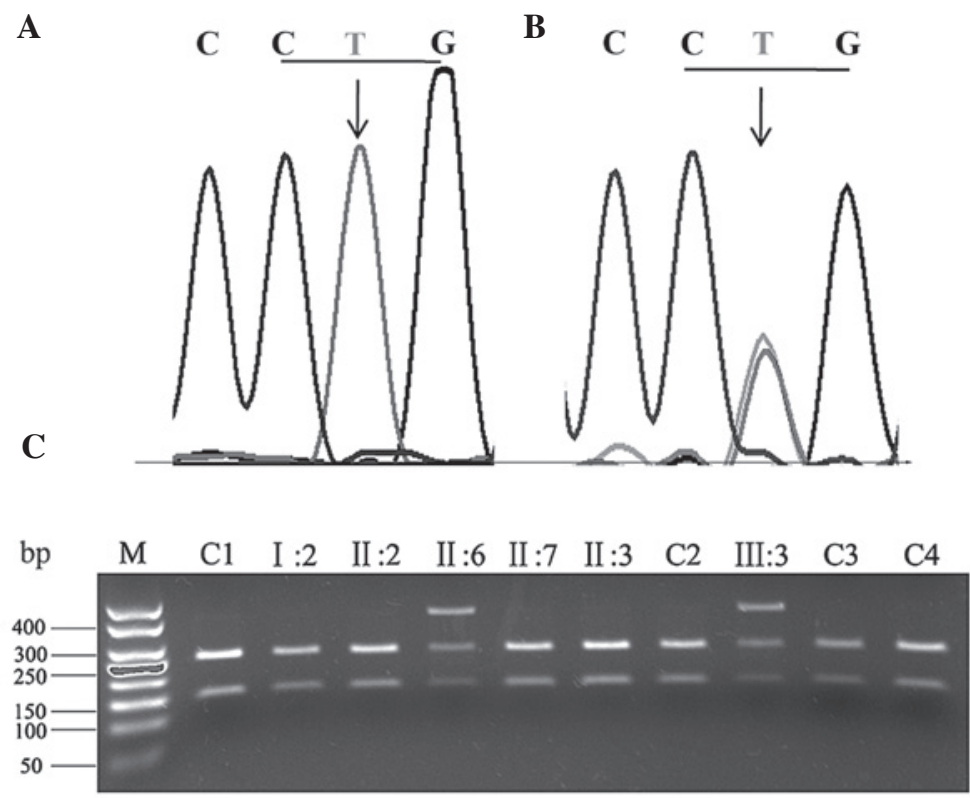

Figure 2. Mutation analysis of keratin 5. Sequence chromatograms of (A) a wild-type allele showing the code for leucine (CTG) at codon 202 and (B) a mutant allele showing a heterozygous $\mathrm{T} \rightarrow \mathrm{A}$ transition that changes leucine at codon 202 to glutamine. (C) Gel patterns showing the products digested with $E c o \mathrm{NI}$ restriction enzyme (affected individuals heterozygous with AT genotype, 401, 256, and 145bp; unaffected individuals with TT genotype, 256 and 145 bp). $\mathrm{M}$, marker; $\mathrm{C} 1, \mathrm{C} 2$ and $\mathrm{C} 3$ represent unrelated healthy individuals.

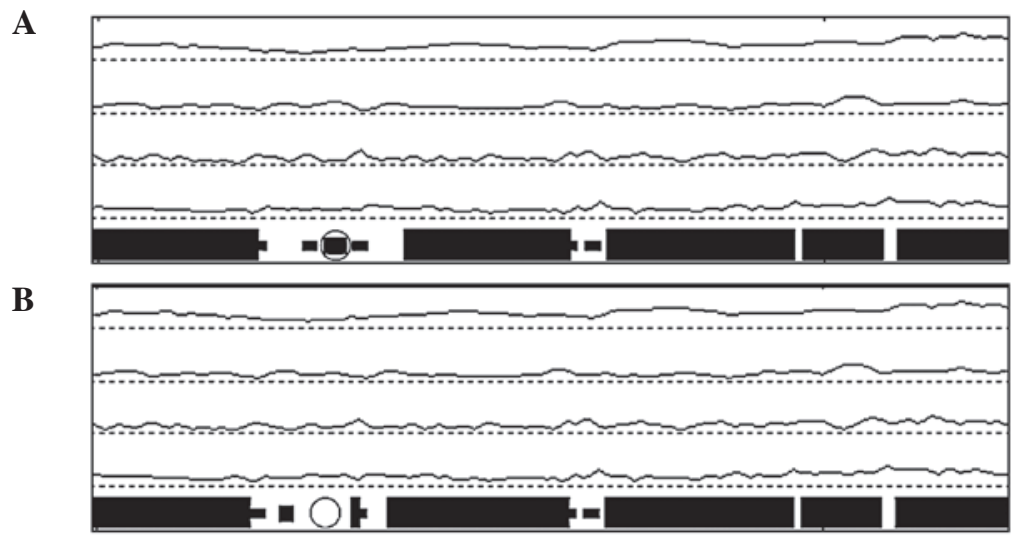

Figure 3. Predicted secondary structures of (A) the wild-type and (B) mutant keratin 5. The target sequences are labeled with black circles. From top to bottom: Helix, sheet, turn, coil.

Prediction of the biophysical properties of the mutant protein. Biophysical predictions of the altered protein were made using bioinformatics tools. In particular, Antheprot 2000 version 5.0 (Institut de Biologie et Chimie des Protéines, Lyon, France) was used for the prediction of secondary structures and hydrophobicity.

\section{Results}

Autosomal dominant pedigree of EBS. Six Chinese patients with EBS were enrolled into the present study, of which two patients (III:3 and II:6) were classified into the EBS-loc subtype based on previously established diagnostic criteria $(4,7)$. The patients had a history suggestive of autosomal dominance. The diagnosis of EBS-loc was confirmed by histopathological examination of the patients' skin, which revealed basal cell cytolysis.
Novel mutation of L202Q in KRT5. In this particular pedigree, the results of direct PCR sequencing in the two affected individuals (III:3 and II:6), in comparison with the wild-type allele (Fig. 2A), revealed a heterozygous $\mathrm{T} \rightarrow \mathrm{A}$ mutation at nucleotide 605 in KRT5 (Fig. 2B). At the protein level, this mutation leads to an amino acid change from leucine to glutamine in codon 202 of exon 2. The results of the PCR-RFLP analysis indicated that this mutation was not present in the unaffected family members or the 100 population controls (Fig. 2C).

Structure predictions. Computer-assisted prediction of the human KRT5 protein was performed in order to obtain a better understanding of the effects of the mutation on its biochemical properties and structure. The results showed that the L202Q mutation caused a variation of the secondary structure with the omission of a $\beta$ sheet (Fig. 3). In addition, the hydrophobicity of the corresponding region (Fig. 4) was decreased. 
Table II. Sequences producing specific alignment of amino acids in keratin 5 .

Species

605 T>A (L202Q)

\section{Dog}

Mouse

Rat

Rabbit

Chicken

Pig

Cattle

Zebrafish

Human (wild-type)

Human (III:3)
VRFLEQQNKVLDTKWTLLQEQGTKTVRQN VRFLEQQNKVLDTKWALLQEQGTKTIKQN VRFLEQQNKVLDTKWALLQEQGTKTVRQN VRFLEQQNKVLDTKWTLLQEQGTKTVRQS VRFLEQQNKVLETKWSLLQEQGMKTVRNN VRFLEQQNKVLDTKWTLLQEQGIKTVRQN VRFLEQQNKVLDTKWTLLQEQGTKTVRQN VRFLEQQNKVLETKWSLLQEQT-TTRSNI VRFLEQQNKVLDTKWTLLQEQGTKTVRQN VRFLEQQNKVLDTKWTQLQEQGTKTVRQN

A protein-protein Basic Local Alignment Search Tool (National Center for Biotechnology Information) search of human keratin 5 amino acid sequence was performed. Proteins having various levels of sequence identity were selected and aligned. Multiple alignments revealed that the mutation site (bold letter) is highly conserved among different species.

A

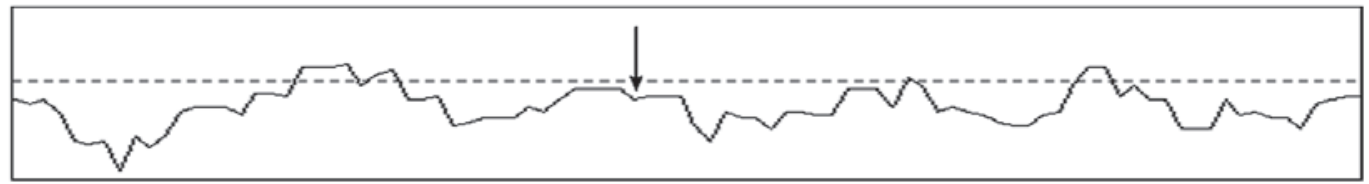

B

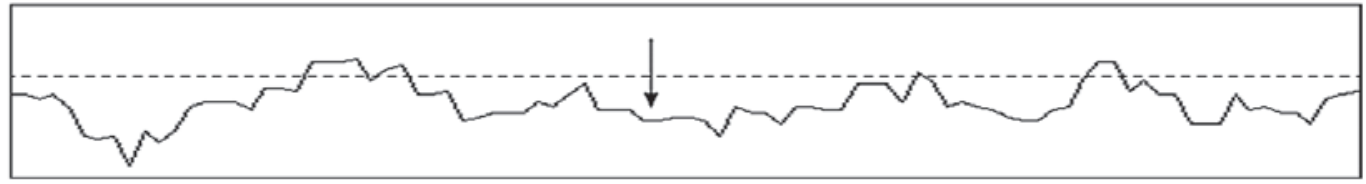

Figure 4. Hydrophobicity plots of (A) wild-type and (B) mutated keratin 5. The x-axis represents the position of amino acids. The y-axis represents the hydrophobicity value in a default window size of 7 . The mutant clearly exhibited lower hydrophilicity in the corresponding region, as compared with the wild-type (indicated by black arrows).

\section{Discussion}

In this pedigree, the diagnosis of EBS-loc with an autosomal dominant transmission mode was made on the basis of the clinical manifestations, family history and histopathological examination of the patients' skin.

Subsequently mutation analysis of the commonest candidate gene KRT5 was conducted using direct PCR sequencing and PCR-RFLP, and a missense mutation (c.605T>A, p.L202Q) was identified in KRT5. Since this mutation cosegregated with the disease in the family and was absent from the population controls, the possibility of a rare polymorphism was excluded, and it was considered as a novel mutation. The mutation replaces leucine with glutamine at the 202th amino acid on position 34 of the 1A helical domain of KRT5, which is highly conserved among other type II keratins (data not shown) and different species including human, dog, rat, mouse, chicken and zebrafish (Table II).

In general, the most severe phenotype of EBS, EBS-DM, has been associated with mutations in more highly conserved helix boundary motifs, including the head of segment $1 \mathrm{~A}$ and end of segment 2B in the rod domains of KRT5, which induce greater keratin aggregation in basal keratinocytes. By contrast, milder phenotypes, such as EBS-loc and EBS-gen, exhibit keratin monofilaments that appear relatively normal ultrastructurally and have been linked to mutations in linker domains and elsewhere in the rod domain of KRT5 (8-10). Consistent with previous reports, the p.L202Q mutation in this pedigree was identified at the end of the 1A domain, a non-helix boundary motif of KRT5, which resulted in the EBS-loc phenotype.

Computer-assisted prediction results indicated that the L202Q mutation would have a significant effect on the secondary structure and hydrophobicity of the protein. As shown in Fig. 4, the mutant appears to be missing a $\beta$ sheet at the mutation site, which may due to the alteration of the polarity of the affected amino acid by the replacement of a non-polar hydrophobic leucine by a polar neutral glutamine. It has been demonstrated that a mutation in the coiled-coil motif of 1A domain of KRT5 often results in a significant distortion of the backbone over a turn, increasing the likelihood of impaired chain aggregation, and hence molecular assembly (11). It was therefore speculated that the omission of a $\beta$ sheet could result in the reduction of hydrophobicity; however, further functional experiments are necessary to explore the underlying mechanisms in detail.

In this pedigree, the individual II:6 suffering from EBS had unaffected parents. There are two possible causes for that: Either the mutation is a de novo mutation or the penetrance of EBS was incomplete. 
The present study reported a novel mutation of EBS in a Chinese pedigree. A previous report has revealed EBS types in Israel that have a unique mutation spectrum and different patterns of inheritance, including a higher incidence of recessive cases than in families from Europe or the USA (12). In addition, the proportion of Japanese patients with EBS with KRT5 mutations is 3-fold higher than those with KRT14 mutations, despite the fact that mutations in these two genes have been reported to be equally prevalent $(13,14)$. Whether the mutation spectrum in Chinese patients with EBS is similar or unique to other ethnic groups remains unclear; therefore, further accumulation of clinical data is required, in order to confirm the spectrum of EBS mutations in China.

\section{Acknowledgements}

The authors would like to thank all the members of the pedigree for their kind cooperation. This study was supported by the National Natural Science Foundation of China (grant no. 81270365$)$.

\section{References}

1. Fine JD, Eady RA, Bauer EA, Briggaman RA, Bruckner Tuderman L, Christiano A, Heagerty A, Hintner H, Jonkman MF, McGrath $\mathrm{J}$, et al: Revised classification system for inherited epidermolysis bullosa: Report of the Second International Consensus Meeting on diagnosis and classification of epidermolysis bullosa. J Am Acad Dermatol 42: 1051-1066, 2000.

2. Coulombe PA, Kerns ML and Fuchs E: Epidermolysis bullosa simplex: A paradigm for disorders of tissue fragility. J Clin Invest 119: 1784-1793, 2009.

3. Fuchs EV: The molecular biology of epidermolysis bullosa simplex. In: Clinical, Epidemiologic, and Laboratory Advances, and the Findings of the National Epidermolysis Bullosa Registry. Fine JD, Bauer EA, McGuire J and Moshell A (eds). Johns Hopkins University Press, Baltimore, pp280-299, 1999.
4. Horn HM and Tidman MJ: The clinical spectrum of epidermolysis bullosa simplex. Br J Dermatol 142: 468-472, 2000.

5. Steinert PM: The two-chain coiled-coil molecule of native epidermal keratin intermediate filaments is a type I-type II heterodimer. J Biol Chem 265: 8766-8774, 1990.

6. Soetens O, Vauloup-Fellous C, Foulon I, Dubreuil P, De Saeger B, Grangeot-Keros L and Naessens A: Evaluation of different cytomegalovirus (CMV) DNA PCR protocols for analysis of dried blood spots from consecutive cases of neonates with congenital CMV infections. J Clin Microbiol 46: 943-946, 2008.

7. Fine JD, Eady RA, Bauer EA, Bauer JW, Bruckner-Tuderman L, Heagerty A, Hintner $\mathrm{H}$, Hovnanian A, Jonkman MF, Leigh I, et al: The classification of inherited epidermolysis bullosa (EB): Report of the third international consensus meeting on diagnosis and classification of EB. J Am Acad Dermatol 58: 931-950, 2008.

8. Liovic M, Stojan J, Bowden PE, Gibbs D, Vahlquist A, Lane EB and Komel R: A novel keratin 5 mutation (K5V186L) in a family with EBS-K: A conservative substitution can lead to development of different disease phenotypes. J Invest Dermatol 116: 964-969, 2001.

9. Murrell DF, Trisnowati N, Miyakis S and Paller AS: The yin and the yang of keratin amino acid substitutions and epidermolysis bullosa simplex. J Invest Dermatol 131: 1787-1790, 2011.

10. Bolling MC, Lemmink HH, Jansen GH and Jonkman MF: Mutations in KRT5 and KRT14 cause epidermolysis bullosa simplex in $75 \%$ of the patients. Br J Dermatol 164: 637-644, 2011.

11. Smith TA, Steinert PM and Parry DA: Modeling effects of mutations in coiled-coil structures: Case study using epidermolysis bullosa simplex mutations in segment 1a of K5/K14 intermediate filaments. Proteins 55: 1043-1052, 2004.

12. Ciubotaru D, Bergman R, Baty D, Indelman M, Pfendner E, Petronius D, Moualem H, Kanaan M, Ben Amitai D, McLean WH, et al: Epidermolysis bullosa simplex in Israel: Clinical and genetic features. Arch Dermatol 139: 498-505, 2003.

13. Yasukawa K, Sawamura D, Goto M, Nakamura H, Jung SY, Kim SC and Shimizu H: Epidermolysis bullosa simplex in Japanese and Korean patients: Genetic studies in 19 cases. $\mathrm{Br}$ J Dermatol 155: 313-317, 2006.

14. Shinkuma S, Natsuga K, Nishie W and Shimizu H: Epidermolysis bullosa in Japan. Dermatol Clin 28: 431-432, 2010. 